\title{
ANTERIOR INTEROSSEOUS NERVE PALSY
}

\author{
C. B. D’A. Fearn and J. W. Goodfellow, Oxford, England
}

From the Nuffield Department of Orthopaedics, Nuffield Orthopaedic Centre, Oxford

Unexplained paralysis of the muscles supplied by the anterior interosseous nerve of the forearm was recorded by Parsonage and Turner (1948), Kiloh and Nevin (1952) and Thomas (1962). In none of the patients they described was the affected nerve explored. Spontaneous recovery occurred in all, but it was long delayed and incomplete. We record here a case of "isolated neuritis of the anterior interosseous nerve" in which exploration revealed a mechanical cause and recovery was rapid and complete following surgical relief of compression.

\section{CASE REPORT}

In August 1962 a boy of nine noticed pain in the front of the left elbow at night, followed by weakness of flexion of the terminal joints of the left thumb and index finger. There was no history of injury. Examination a month after the onset of symptoms revealed 10 degrees limitation of flexion at the elbow and a point of tenderness in the lower part of the cubital fossa. The strength of flexor pollicis longus muscle power was 4 (Medical Research Council grading). The flexor digitorum profundus of the index finger was power 3 , and that of the middle finger 4. There was full power in the other muscles of the limb and no alteration of cutaneous sensation. After wearing a sling for three weeks there was some improvement but when movement at the elbow was again allowed the weakness in the long flexors became more obvious, and attacks of pins-and-needles in the index finger occurred.

He was admitted to the Nuffield Orthopaedic Centre in November 1962 with complete paralysis of flexor pollicis longus muscle and marked weakness of the long flexor of the index finger. It was decided to explore the cubital fossa. At operation a longitudinal incision was made in the midline of the anterior surface of the forearm from the cubital fossa distally. No abnormality was found at the elbow. The median nerve was traced to the point at which the anterior interosseous nerve branched from the main trunk. One centimetre below this level a glistening crescentic fibrous band was seen to lie across the median nerve and its anterior interosseous branch (Fig. 1). The band was continuous distally with the fibrous aponeurosis on the deep surface of the superficial (humeral) head of pronator teres. Proximally the fibres of the band fanned out and blended with the insertion of the brachialis muscle. Supination of the forearm with the elbow extended tightened the band which then pressed on the anterior interosseous and median nerves. Before dividing the band, both nerves were dissected more distally and the main trunk was seen to pass between the superficial (humeral) and deep (ulnar) heads of pronator teres into the forearm. The fibrous band was then excised. Electrical stimulation of the anterior interosseous nerve was shown to produce flexion of the terminal phalanx of the thumb but failed to produce movement in the index finger.

Six weeks after the operation recovery had taken place in the flexor pollicis longus which was strength 4 . The long flexor of the index finger was only strength 2 . Three months later recovery was complete in both these muscles and in the long flexor of the middle finger.

\section{DISCUSSION}

Parsonage and Turner (1948) in their review of 136 cases of muscular paralysis about the shoulder recorded five patients in whom there was concurrent paralysis of flexor pollicis longus and flexor digitorum profundus to the index finger. They also described one patient who had an isolated paralysis of these muscles. 
Kiloh and Nevin (1952) described two patients in whom the anterior interosseous nerve alone was affected. In the first there was complete paralysis of the right flexor pollicis longus and weakness of the long flexors of the index and middle fingers. With conservative treatment there was some improvement after a year but after two years the thumb and index fingers were still weak. In their second case the left flexor pollicis longus alone was affected, being completely paralysed for ten months. Five months later, after treatment with galvanic stimulation, the strength was $4-$. Both their patients complained of pain in the forearm at the time of the onset of paralysis.

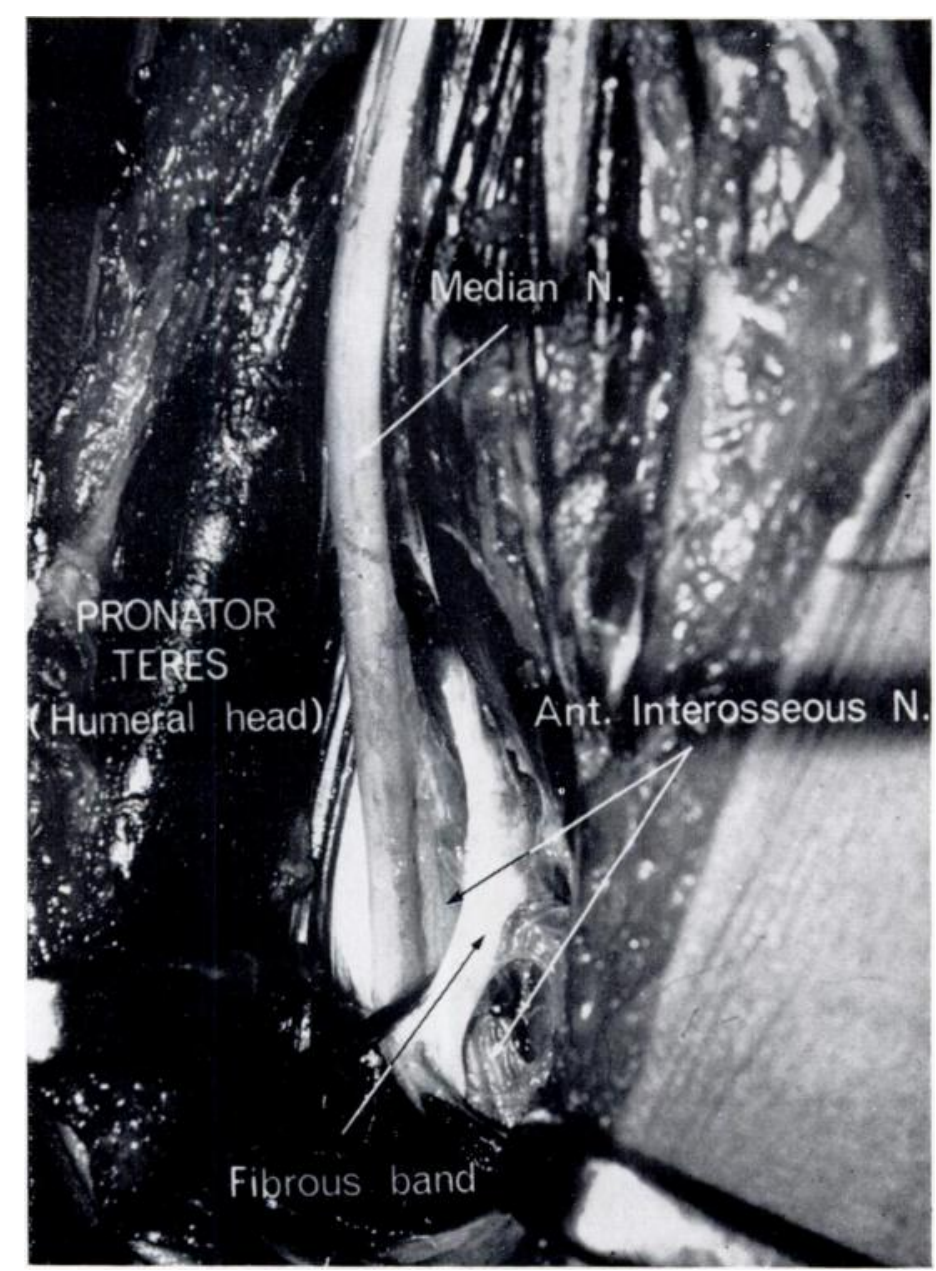

Fig. 1

Photograph at operation of the fibrous band arching over the left median nerve and its anterior interosseous branch.

Thomas (1962) described two more patients. In one the anterior interosseous nerve paralysis developed suddenly six weeks after a road accident in which the patient had suffered some superficial wounds about the elbow. In the second patient a complete paralysis of flexor pollicis longus was suddenly noticed without any associated pain and examination revealed no other paresis or altered sensation.

Thomas pointed to the clinical features which his patients and those of Kiloh and Nevin shared. He believed them to be examples of a syndrome characterised by sudden awareness of paresis with transient forearm pain either before or immediately after paralysis of the flexor 
pollicis longus muscle, variable paralysis of the lateral half of the flexor digitorum profundus, and slow and incomplete recovery.

There seems little doubt that in our patient the paralysis was due to compression of the nerve by the fibrous band. This explanation is supported by the transient improvement observed before operation as the result of relative immobilisation of the elbow in a sling and by the rapid and complete recovery which followed surgical division of the offending structure.

We are not certain which anatomical term to apply to the fibrous band. It was not the ulnar head of the pronator teres, for that structure was identified separately. Nor did it seem to fit the description of the fibrous arch which joins the two heads of flexor digitorum sublimis, for it blended distally with the humeral head of pronator teres. In an effort to understand the anatomy we dissected twelve cubital fossae in six post-mortem subjects and found much variation in the mode of origin of both muscles. The pronator teres varies in two respects: firstly, in the state of the ulnar head, which is sometimes fleshy and sometimes tendinous; and secondly, in the degree of attachment of the humeral head to the underlying flexor digitorum sublimis muscle. It is this latter variation that makes precise description difficult, for in several subjects the fibrous arch of flexor digitorum sublimis blended distally with both heads of pronator teres. The conventional anatomical description of the origins of these muscles is an over-simplification. We must add that there is great variation in the amount of fibrous tissue uniting the two muscles in the region of the median nerve.

The distribution of the paralysis in our patient, as in those previously recorded, suggests that in every case the nerve palsy was complete. In all there was complete paralysis of flexor pollicis longus, the only muscle solely innervated by the anterior interosseous nerve that can be readily tested clinically. The degree of paresis of the radial part of the flexor digitorum profundus varied from case to case, but this muscle has a double innervation, and it is to be expected that the contribution of the anterior interosseous nerve might vary from patient to patient.

Brooks (1963) in a recent review of nerve compression syndromes has pointed to the importance of their early recognition while changes in nerve and muscle are still reversible and treatment a relatively simple matter. We suggest that the "anterior interosseous nerve syndrome" is no exception to the rule that one should be wary of ascribing paralysis in the distribution of a peripheral nerve to a hypothetical "neuritis," and that a search for a mechanical cause may be rewarding.

\section{SUMMARY}

An isolated palsy of the anterior interosseous nerve of the forearm is described in a boy aged nine. It was cured by surgical division of a constricting fibrous band in the forearm.

We are grateful to Dr Aloysio Campos da Paz for the photograph and to Professor D. M. Pryce of St Mary`s Hospital Medical School, who made available material for dissection.

\section{REFERENCES}

Brooks, D. M. (1963): Nerve Compression Syndromes. Journal of Bone and Joint Surgery, 45-B, 445.

KILOH, L. G., and NeVIN, S. (1952): Isolated Neuritis of Anterior Interosseous Nerve. British Medical Journal, i, 850.

Parsonage, M. J., and Turner, J. W. (1948): Aneuralgic amyotrophy: Shoulder-girdle Syndrome. Lancet, i, 173.

Тномаs, D. F. (1962): Kiloh-Nevin Syndrome. Journal of Bone and Joint Surgery, 44-B, 962. 